RESEARCH ArTiCLE

Published December 30, 2020

\title{
Evaluation of Six Weekly Oral Fecal Microbiota Transplants in People with HIV
}

\author{
AUTHORS \\ Netanya S. Utay, $\mathrm{MD}^{1,2}$, Ana N. Monczor, $\mathrm{MD}^{3}$, Anoma Somasunderam, $\mathrm{PhD}^{1}$, Sofia Lupo, $\mathrm{MD}^{3}$, \\ Zhi-Dong Jiang, $\mathrm{MD}$, $\mathrm{DrPh}^{4}$, Ashley S. Alexander, $\mathrm{MHSA}^{2}$, Malcolm Finkelman, $\mathrm{PhD}^{5}$, Karen J. \\ Vigil, $\mathrm{MD}^{3}$, Jordan E. Lake, MD, $\mathrm{MSc}^{3}$, Blake Hanson, $\mathrm{PhD}^{4}$, Herbert L. DuPont, $\mathrm{MD}^{2,4}$, Roberto \\ C. Arduino, $\mathrm{MD}^{3}$
}

\section{AFFILIATED INSTITUTIONS}

${ }^{1}$ Division of General Medicine, Department of Internal Medicine, McGovern Medical School at The University of Texas Health Science Center at Houston, Houston, Texas

${ }^{2}$ Kelsey Research Foundation, Houston, Texas

${ }^{3}$ Division of Infectious Diseases, Department of Internal Medicine, McGovern Medical School at The University of Texas Health Science Center at Houston, Houston, Texas

${ }^{4}$ School of Public Health at The University of Texas Health Science Center at Houston, Houston, Texas

${ }^{5}$ Associates of Cape Cod Inc., Falmouth, Massachusetts

\section{CORRESPONDING AUTHOR}

Netanya S. Utay

University of Texas Health Science Center at Houston

6431 Fannin Street, MSB 1.122

Houston, TX 77030

netanya.s.utay@uth.tmc.edu

Phone: 713-500-6810

\section{DOI}

10.20411/pai.v5i1.388

\section{SUGGESTED CITATION}

Utay NS, Monczor AN, Somasunderam A, Lupo S, Jiang ZD, Alexander AS, Finkelman M, Vigil KJ, Lake JE, Hanson B, DuPont HL, Arduino RC. Evaluation of Six Weekly Oral Fecal Microbiota Transplants in People with HIV. Pathogens and Immunity. 2020;5(1):364-381. PubMed PMID. doi: $\underline{10.20411 / \text { pai.v5i1.388 }}$ 


\section{ABSTRACT}

Background: Reduced microbiota diversity (dysbiosis) in people with HIV (PWH) likely contributes to inflammation, a driver of morbidity and mortality. We aimed to evaluate the safety and tolerability of 6 weekly oral fecal microbiota transplants (FMT) administered to reverse this dysbiosis.

Methods: Six PWH on suppressive antiretroviral therapy (ART) received 6 weekly doses of lyophilized fecal microbiota product from healthy donors. Shotgun sequencing on stool before, after last FMT, and 20 weeks thereafter was performed. Inflammation and gut permeability biomarkers were measured.

Results: Median age at week 0 was 39 years, CD4 ${ }^{+} \mathrm{T}$ cell count 496 cells/ $\mathrm{mm}^{3}$, HIV RNA levels $<20$ copies/mL. FMT was safe and well-tolerated. $\alpha$ diversity increased in 4 participants from weeks 0 to 6 , including the 3 with the lowest $\alpha$ diversity at week 0 . At week 26, $\alpha$ diversity more closely resembled week 0 than week 6 in these 4 participants. Metagenomic analysis showed no consistent changes across all participants. One participant had high gut permeability and inflammation biomarker levels and low $\alpha$ diversity that improved between weeks 0 and 6 with a shift in distribution.

Conclusions: Weekly FMT was safe and well-tolerated. $\alpha$ diversity increased in participants with the lowest baseline $\alpha$ diversity during the treatment period. Future randomized, controlled trials of FMT should consider evaluating PWH with greater inflammation, gut damage, or dysbiosis as this population may be most likely to show a significant response.

ClinicalTrials.gov Identifier: NCT03329560

Keywords: HIV, microbiome, fecal microbiota transplant, inflammation

\section{INTRODUCTION}

People with HIV (PWH) taking suppressive antiretroviral therapy (ART) remain at increased risk of cardiovascular events, malignancies, and other comorbidities, likely due to persistent inflammation [1]. A key driver of this inflammation is increased translocation of microbial products across a permeable gut barrier [2]. In HIV, CD4 ${ }^{+} \mathrm{T}$ cells are rapidly depleted from the intestinal epithelial barrier, with destruction of tight junctions, enterocyte death, decreased IgA and mucus production, and dysfunctional macrophages. Levels of biomarkers of increased gut permeability, microbial translocation, and systemic inflammation remain increased despite the long-term treatment of HIV infection, and these biomarkers predict morbidity and mortality [ $\underline{3}-\underline{5}]$.

Many studies have demonstrated that PWH have an abnormal gut microbiome, characterized by decreased $\alpha$ diversity (dysbiosis) and a shift toward more pro-inflammatory Proteobacteria and fewer anti-inflammatory bacteria that produce short chain fatty acids (SCFAs) []. Erysipelotrichaceae, Enterobacteriaceae, Desulfovibrionaceae, and Fusobacteria are increased, whereas Lachnospiraceae, Ruminococceae, Bacteroides, and Rikenellaceae are decreased in the microbiomes of PWH. This abnormal microbiome may perpetuate gut damage and chronic systemic inflammation [7]. Some studies have found that alterations in gut flora, particularly increases in Prevotella and decreases in Bacteroides, only affect men who have sex with men (MSM) [ $\underline{8}-\underline{11}]$. However, Proteobacteria, Desulfovibrio, Enterobacteriaceae, Fusobacteria, Ruminococcaceae, and Lachno- 
spiraceae do not seem to differ between MSM and non-MSM participants []]. Nonetheless, these findings suggest that sexual activity needs to be considered when conducting microbiome studies.

Fecal microbiota transplant (FMT) is an emerging treatment modality and is in the guidelines for treatment of refractory Clostridioides difficile infection [12]. FMT is being explored for inflammatory bowel disease (IBD), Alzheimer's disease, non-alcoholic fatty liver disease, and other conditions. A one-time FMT delivered by colonoscopy was safe in $6 \mathrm{PWH}$ and showed minimal shifts in the microbiota [13]. However, in other chronic conditions such as IBD, multiple FMTs may be more effective $[\underline{14}, \underline{15}]$.

We developed an oral, lyophilized, encapsulated FMT product derived from stool of healthy donors [16]. This product has $84 \%$ efficacy in treating refractory $C$. difficile infection with excellent tolerability. We hypothesized that 6 weekly FMTs with this lyophilized product delivered by the oral route would be safe, increase $\alpha$ diversity (i.e., decrease dysbiosis) and shift the microbiome in 6 MSM with HIV on suppressive ART.

\section{METHODS}

\section{Study Design}

Participants were eligible if they were MSM, HIV positive, on continuous ART for $\geq 24$ weeks, with $\mathrm{CD} 4^{+} \mathrm{T}$ cell count $>350$ cells $/ \mathrm{mm}^{3}$, HIV RNA levels $<20$ copies $/ \mathrm{ml}$ for $\geq 12$ weeks, and absolute neutrophil count $\geq 1000$ cells $/ \mathrm{mm}^{3}$. Participants were excluded if they commenced ART during acute or early HIV infection; had hepatitis B or C (detectable HCV RNA level) co-infection; received antibiotics, investigational therapies, or vaccines within 60 days; had serious illness within 30 days, cirrhosis, chronic gastrointestinal disease, diabetes mellitus, acute or persistent diarrhea within 60 days; or used immunosuppressive drugs, immune modulators, antineoplastic agents, prebiotics or probiotics for $>3$ consecutive days within 60 days. We recruited 6 participants from Thomas Street Health Center in Houston, Texas. This study was reviewed and approved by the UTHealth Committee for the Protection of Human Subjects. All participants signed an informed consent form. Human experimentation guidelines of the United States Department of Health and Human Services and UTHealth were followed in the conduct of clinical research. ClinicalTrials.gov Identifier: NCT03329560.

\section{Study Intervention}

The encapsulated lyophilized FMT product, PRIM-DJ2727, was prepared as previously described [16]. Donors were healthy men age 56 to 68 years old with $\mathrm{BMI}<30 \mathrm{~kg} / \mathrm{m}^{2}$ and no personal or family history of disease suspected to be transmitted by the microbiome. Each participant received the investigational product derived from 150 grams of stool (lyophilized to approximately $2.25 \mathrm{~g}$ ) from 1 of 3 donors, weekly for 6 weeks. Participants fasted except for water for 8 hours before FMT. They took the product under supervision and were monitored for 1.5 hours after ingestion. Serum and EDTA plasma and stool by self-collected rectal FLOQSwabs were obtained from the participants. Transient elastography with controlled attenuation parameter (Fibroscan $\left.{ }^{\circledast}\right)$ was performed at weeks 0 and 26 . 


\section{Isolation of DNA from Stool}

DNA from the stool samples was isolated using the QIAamp Biostic Bacteremia DNA kit (Qiagen, Germantown, MD). DNA concentration was determined using Nanodrop (Thermo Scientific, Waltham, MA).

\section{Biomarker Measurements}

Biomarkers were measured on EDTA plasma or serum. Intestinal fatty acid binding protein (I-FABP) was measured using the DuoSet ELISA Kit; sCD14 and IL-6 were measured by ELISA using Quantikine kits; sCD163 and sTNFRII were measured using Luminex high-performance assays (all R\&D systems, Minneapolis MN). Serum (1,3)- $\beta$-D-glucan was measured using the Fungitell kit (Associates of Cape Cod, Inc., Falmouth, MA). Other inflammation biomarkers were measured using the Luminex platform (Bio-Plex Pro ${ }^{\text {та }}$ Human Inflammation Panel 1, BioRad Laboratories, Inc., Hercules, CA).

\section{Sequencing}

DNA was analyzed by shotgun sequencing to achieve strain level resolution of the microbiome (CosmosID, Rockville, MD). Fragment libraries were generated for the DNA using the ThermoFisher IonXpress Plus Fragment Library kit according to the manufacturer's instructions. The libraries were sequenced on an Ion S5XL sequencer to generate 200bp sequences. The depth of the reads in the samples averaged $22.9 \mathrm{M}$, with a standard deviation of $6.3 \mathrm{M}$, a range of $15.8-35.3 \mathrm{M}$, and a median of $21.1 \mathrm{M}$ reads. 546 bacterial species and 765 bacterial strains were identified with high confidence. These organisms met a filtering threshold that is based on internal statistical scores determined by analyzing a large number of diverse metagenomes [17].

\section{Metagenomic Profiling}

Unassembled sequencing reads were directly analyzed by the CosmosID bioinformatics platform (CosmosID Inc., Rockville, MD), as previously described [18-21] for multi-kingdom microbiome analysis and quantification of an organism's relative abundance. The system utilizes curated genome databases and a high-performance data-mining algorithm that rapidly disambiguates hundreds of millions of metagenomic sequence reads into the discrete microorganisms engendering the particular sequences.

\section{Functional Analysis}

For functional analysis of the metagenomic data, paired-end reads of the sequencing data were trimmed using BBDuk (https://jgi.doe.gov/data-and-tools/bbtools/) with the parameters minlen=25 qtrim=rl trimq=20. MegaHit [22] was used to construct assemblies from filtered raw reads of metagenomes using 77, 99, 127 values for K-mer size parameter. Each assembled metagenome was sent to Prokka [23] to predict ORF-based protein coding genes and assign functions to the predicted genes in text and GenBank formats. Sequences of the genes, in Fasta format, were used to map to the raw FastQ files using BBMap with allowed fragments per kilobase of gene per million (FPKM) parameter, which produced sample-wise output in text format. Sequences of predicted protein coding genes, identified in Fasta format from the Prokka, were sent to InterProScan for assignment of KEGG pathways and GO processes, including 22 pathways and 143 processes. FPKM values of every protein/gene assigned to each of the KEGG pathway or GO pro- 
cess were derived from mapping the gene to the FPKM list of the genes identified in the previous step. To obtain abundance of each of the KEGG pathways and GO processes, the FPKM values of genes in a pathway or process were summed up and considered as the abundance of the pathway and process, respectively.

\section{Statistical Methods}

Data between time points were compared using Wilcoxon matched-pairs signed rank test and between PWH and donors using Student's t test. Spearman correlation coefficient was used for correlations using GraphPad Prism version 6.2 (San Diego, CA).

\section{RESULTS}

\section{Baseline Characteristics}

Median age was 39 (range 32-56) years, median BMI $27.7 \mathrm{~kg} / \mathrm{m}^{2}$ (range 25.7-31.8), and median $\mathrm{CD}^{+}$T cell count 496 (range 393-1029) cells $/ \mathrm{mm}^{3}$; all participants had HIV RNA levels $<20$ copies/mL (Table 1). Four participants were Hispanic/Latino, 1 was White non-Hispanic/Latino, and 1 was Black/African American. Median time since diagnosis was 5.5 (range 2-35) years, and median time on ART was 5.5 (range 2-7 years) years. No participant consumed alcohol more than twice a week.

Table 1. Baseline Characteristics

\begin{tabular}{|c|c|c|c|c|c|c|}
\hline & PID1 & PID2 & PID3 & PID4 & PID5 & PID6 \\
\hline Age (years) & 47 & 40 & 56 & 33 & 37 & 32 \\
\hline Race & White & White & Black & White & White & White \\
\hline Ethnicity (Hispanic) & No & Yes & No & Yes & Yes & Yes \\
\hline CD4 (cells $\left./ \mathrm{mm}^{3}\right)$ & 1029 & 505 & 510 & 471 & 393 & 487 \\
\hline CD8 (cells $\left./ \mathrm{mm}^{3}\right)$ & 2587 & 853 & 857 & 825 & 837 & 548 \\
\hline $\mathrm{CD} 4 / \mathrm{CD} 8$ & 0.29 & 0.59 & 0.60 & 0.57 & 0.47 & 0.89 \\
\hline Years since diagnosis & 12 & 4 & 35 & 7 & 3 & 2 \\
\hline Years on $\mathrm{ART}^{1}$ & 7 & 4 & 7 & 7 & 3 & 2 \\
\hline ART regimen & $\begin{array}{c}\text { TAF/ } \\
\text { FTC/ } \\
\text { cEVG }^{2}\end{array}$ & $\begin{array}{l}\text { TAF/ } \\
\text { FTC/ } \\
\text { cEVG }\end{array}$ & $\begin{array}{l}\mathrm{ABCl} \\
3 \mathrm{TC} / \\
\mathrm{DTG}^{3}\end{array}$ & $\begin{array}{c}\text { TAF/ } \\
\text { FTC } \\
+ \text { DTG } \\
\end{array}$ & $\begin{array}{l}\mathrm{ABC} / \\
3 \mathrm{TC} / \\
\mathrm{DTG}\end{array}$ & $\begin{array}{l}\text { TAF/ } \\
\text { FTC/ } \\
\text { cEVG }\end{array}$ \\
\hline Weight $(\mathrm{kg})$ & 86 & 93 & 93 & 84 & 77 & 80 \\
\hline Waist $(\mathrm{cm})$ & 99 & 108 & 105 & 110 & 97 & 99 \\
\hline $\begin{array}{l}\text { Hepatic steatosis } \\
(\mathrm{dB} / \mathrm{m})\end{array}$ & 275 & 280 & 212 & 345 & 298 & 262 \\
\hline
\end{tabular}

${ }^{1} \mathrm{ART}=$ Antiretroviral therapy; ${ }^{2} \mathrm{TAF}=$ Tenofovir alafenamide; FTC=Emtricitabine; $\mathrm{cEVG}=$ cobicistat/elvitegravir; ${ }^{3} \mathrm{ABC}=$ Abacavir; $3 \mathrm{TC}=$ Lamivudine; $\mathrm{DTG}=$ Dolutegravir 


\section{Safety and Tolerability}

Overall, PRIM-DJ2727 was safe and well-tolerated. No participants developed fever or tachycardia. Participant identification (PID) 2 developed grade 1 abdominal pain at week 1 and grade 1 nausea at week 2. PID3 had mild bloating on 1 day each in weeks 1,2 , and 4, and moderate bloating on the last 2 days of week 6 . No other study drug-related adverse events were noted.

\section{HIV Parameters}

$\mathrm{CD}^{+} \mathrm{T}$ cell counts did not change significantly between weeks 0 and 6 (496 [range 393-1029] vs 463 [range 329-900] cells $\left./ \mathrm{mm}^{3} ; P=0.56\right)$ or weeks 6 and 26 (463 [range 329-900] vs 526 [range 329-1279]; $P=0.13$ ) cells $/ \mathrm{mm}^{3}$ (Figure $1 \mathrm{~A}$ ). $\mathrm{CD} 8^{+} \mathrm{T}$ cell counts tended to decrease between weeks 0 and 6 (845 [range 548-2587] vs 717 [range 512-2272] cells $/ \mathrm{mm}^{3} ; P=0.06$ ) but did not change significantly between weeks 6 and 26 (717 [range 512-2272] vs 665 [range 558-3014]; $P=0.56$ ) (Figure 1B). CD4/CD8 ratios did not change significantly between weeks 0 and 6 ( 0.58 [range $0.40-0.89$ ] vs 0.61 [range $0.40-0.98$ ]; $P=0.31)$ or between weeks 6 and 26 ( 0.61 [range $0.40-0.98$ ] vs 0.71 [range 0.42-0.96]; $P=0.22$ ) (Figure 1C).

Two participants maintained HIV RNA levels $<20$ copies/mL at weeks 0,6 , and 26 , whereas the other participants developed detectable HIV RNA levels (Figure 1D). Three participants subsequently had undetectable levels. Two of these participants had 34-day gaps between ART refills during the study. PID6 stopped ART at week 6 but subsequently resumed ART with HIV RNA level of 130 copies/mL at week 26.

A

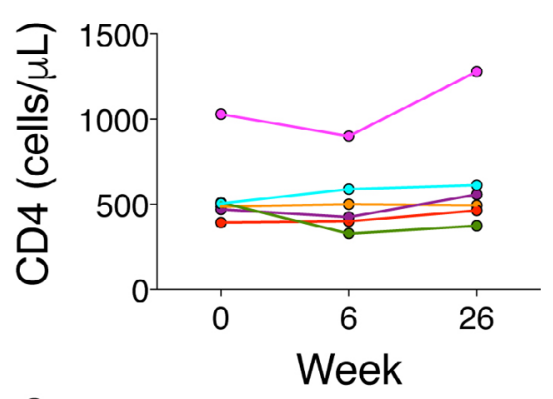

C

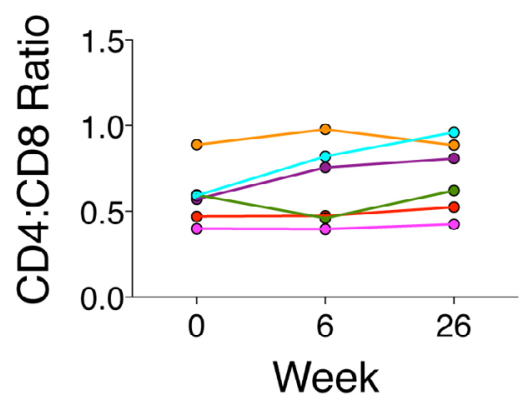

B

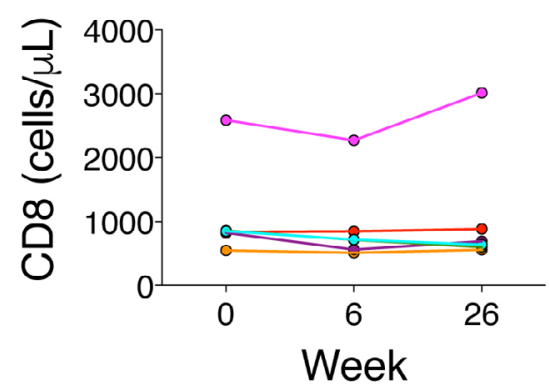

D

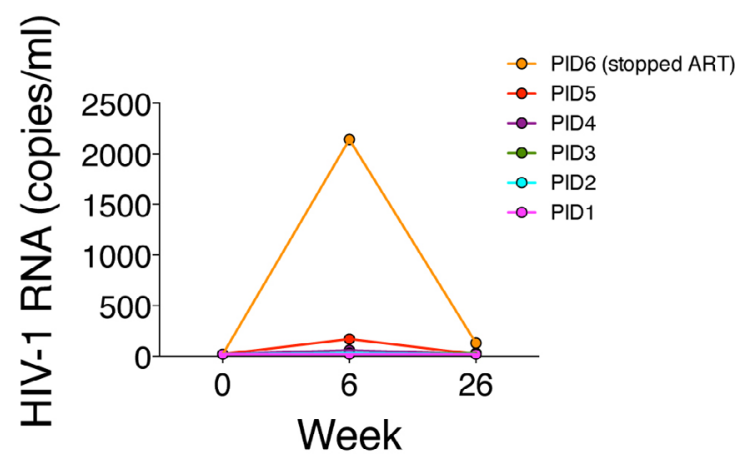

Figure 1. Changes in HIV parameters during treatment period (weeks 0 to 6) and after 20 weeks of followup without FMT. 


\section{Microbiome Effects}

Mean $\alpha$ diversity by observed species index did not materially change from weeks 0 to 6 (61.2 to 70.2, $P=0.29$; Figure 2A) or from weeks 6 to 26 (70.2 to 52.2, $P=0.21)$. $\alpha$ diversity increased in 4 participants (67\%) from weeks 0 to 6 , including the 3 with the lowest $\alpha$ diversity at week 0 (Figure $2 A)$. At week 26, $\alpha$ diversity more closely resembled week 0 than week 6 in 3 of these 4 participants.

Microbiome distribution by Bray-Curtis principal component analysis (PCA) shifted towards the donors' distribution in 3 participants (50\%) at week 6, all of whom had an increase in $\alpha$ diversity between weeks 0 and 6, but shifted away by week 26 (Figure 2B). Microbiome distribution varied across participants (Figure 3). There were no statistically significant changes in any specific strains, species, genera, or families, or in phage distribution.
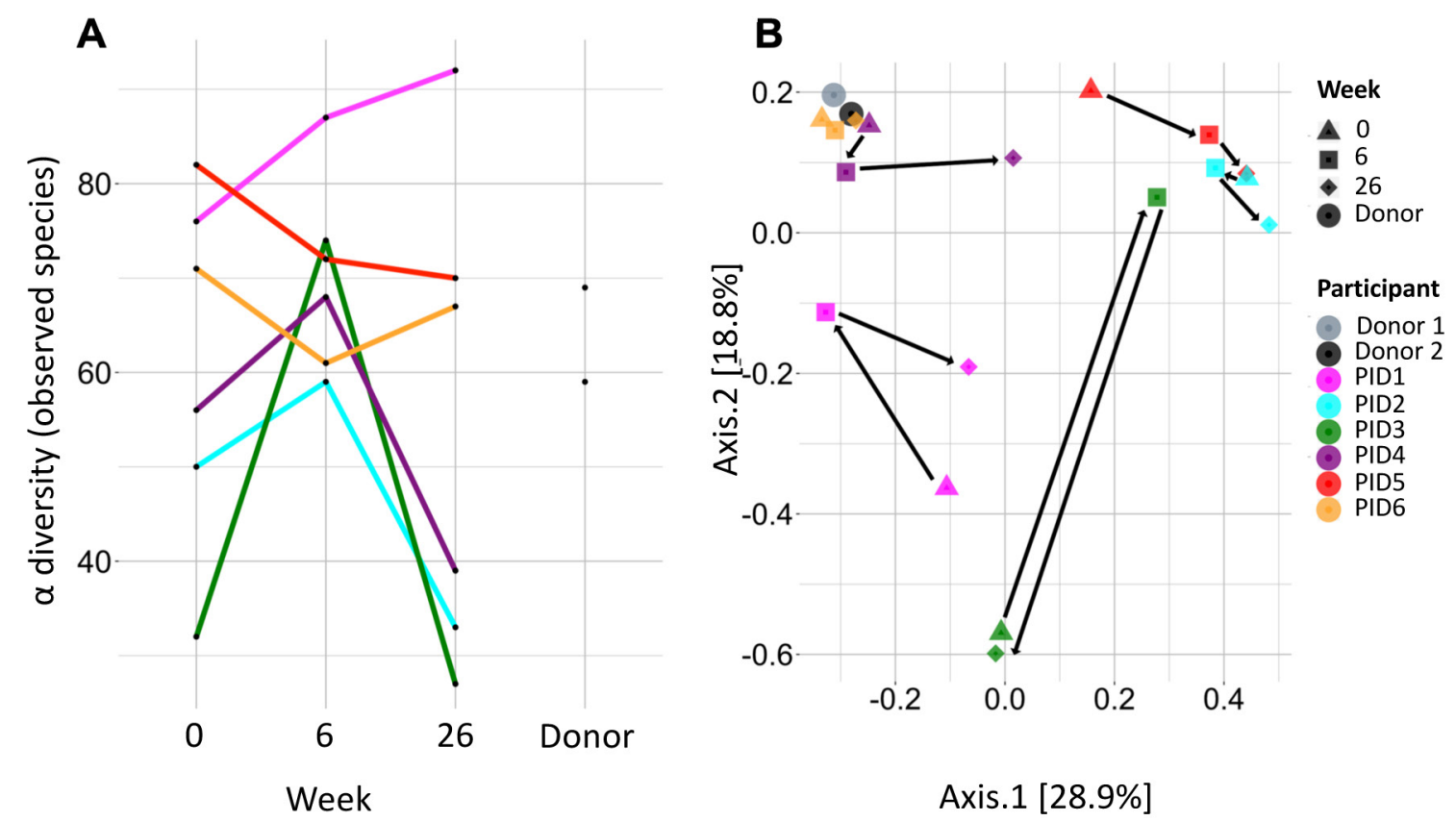

Figure 2. A) $\alpha$ diversity by observed species in each recipient before fecal microbiota transplant (FMT; week 0), after 6 weekly oral FMTs (week 6), and 20 weeks after last FMT (week 26), as well as in donors. B) Microbiome distribution by Bray-Curtis principal component analysis in each recipient at weeks 0,6 , and 26 and in the donors. Circles on the graph reflect the donors (light and dark gray). Triangles indicate week 0 , squares indicate week 6 , and diamonds indicate week 26 ; each is color-coded for participant according to the color in the circle in the key. 

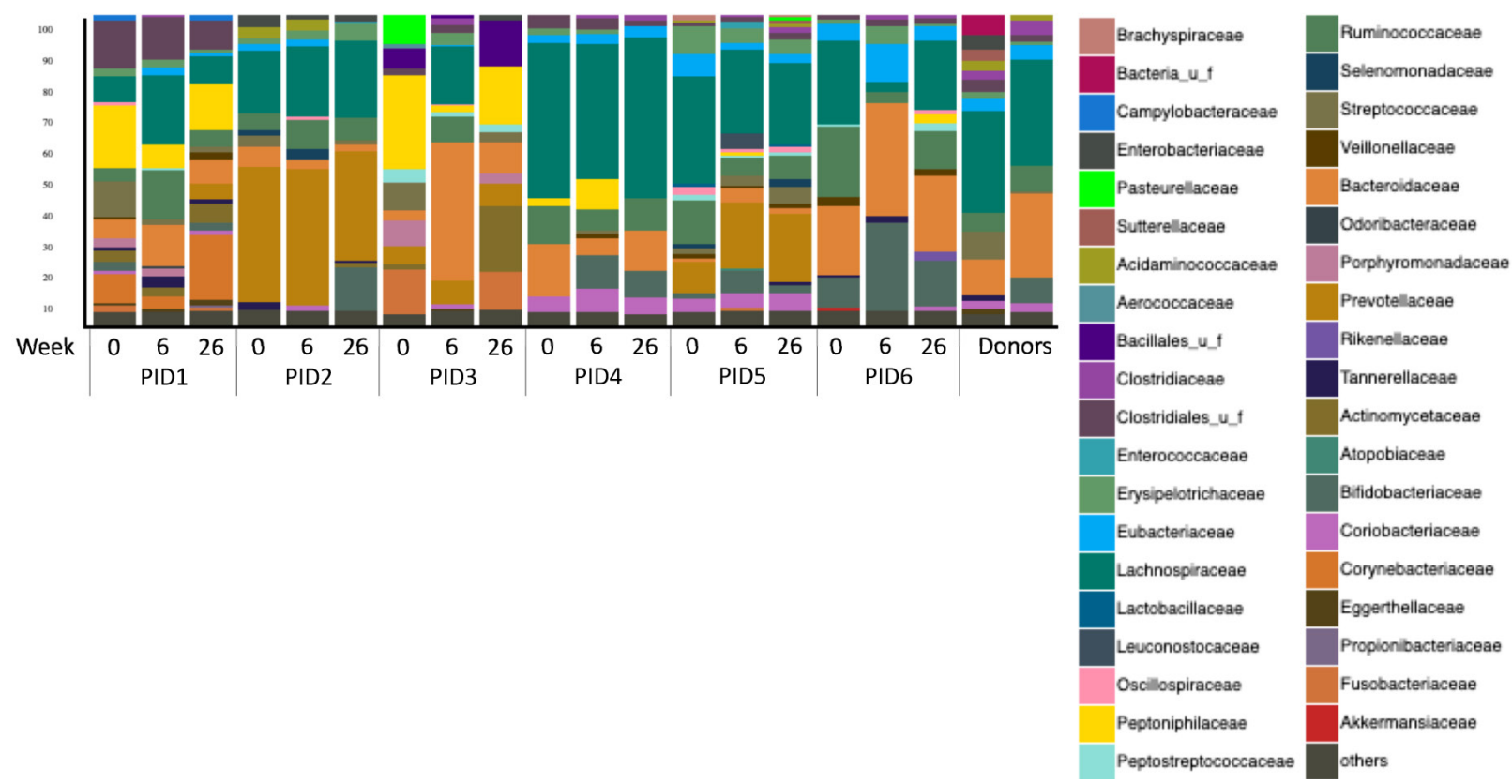

Figure 3. Microbial composition of the participants and donors at the family level. Families with abundance $<5 \%$ were grouped into "others" category.

PID3, who had HIV for $>35$ years and had been taking ART for 7 years, had persistent constipation that resolved between weeks 0 and 6 with a dramatic shift in distribution. Seventeen strains increased by $>1 \%$ relative abundance (Figure $4 \mathrm{~A}$ ), including several Bacteroides, Lachnospiraceae, and Ruminococcus strains, all of which synthesize SCFAs and convert primary into secondary bile acids [24-27]. Nineteen strains decreased by $>1 \%$ relative abundance, including numerous oral microbes (Figure 4B). Of note, PID3 also had increases in numerous bacteriophages, including Yersinia phage L-413C, Enterobacteria phage P4, and Escherichia virus P2, with decreases in Haemophilus phage Hp1 (data not shown). His constipation recurred by week 26 with regression of his microbiome to approximate its baseline distribution and $\alpha$ diversity, although the phage changes persisted.

\section{Metagenomics}

Next we evaluated changes in gene expression pathways using metagenomics. Ribosome pathways, reflecting protein synthesis, tended to decrease in participants during the treatment period $(P=0.09)$, with decreases in 5 of 6 participants (83\%; Supplementary Figure 1A). The 2 donors had higher L-arabinose isomerase activity, reflecting carbohydrate degradation, (Supplementary Figure 1B) and pentose and glucuronate interconversions (Supplementary Figure 1C), than all recipients at all time points. In PID3, several pathways increased between weeks 0 and 6: 1) defense response to Gram-negative bacterium; 2) lipopolysaccharide biosynthesis; 3) spore germination; 4) peptidase activity; 5) porphyrin and chlorophyll metabolism; 6) inositol phosphate metabolism; 7) glycine, serine, and threonine metabolism; and 8) glycerophospholipid metabolism (data not shown). Pathways that decreased during the treatment period in PID3 include 1) cell septum assembly; 2) pathways of energy generation and metabolism (arginine biosynthesis, fumarate 

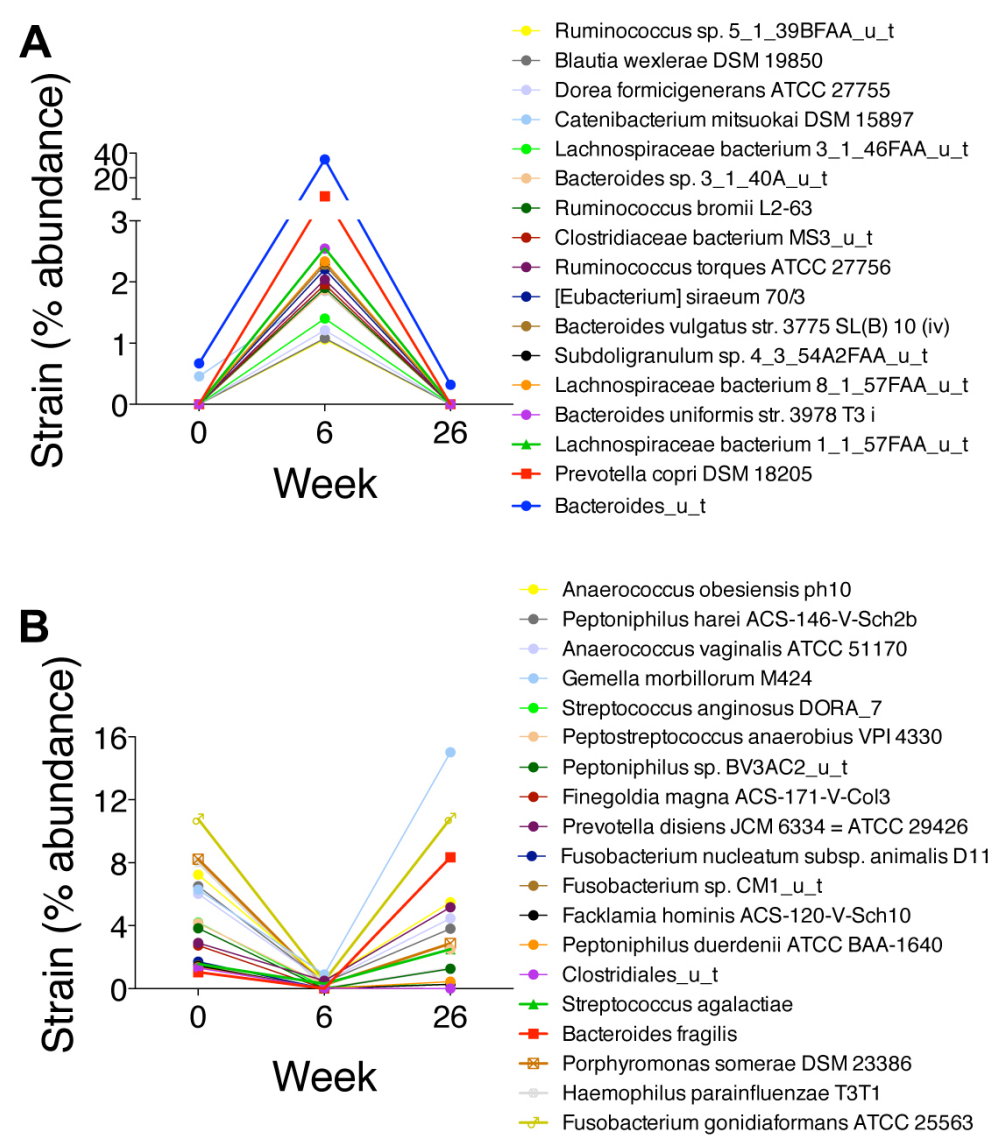

Figure 4. Changes in the microbiota at the strain level in PID3, the participant who had the highest zonulin, intestinal fatty acid binding protein (I-FABP), soluble TNF receptors I and II, soluble TNF receptor superfamily 8 (sCD30), and osteopontin levels and had a dramatic shift in distribution. A) Increases in strain abundance by at least 1\%. B) Decreases in strain abundance by at least $1 \%$.

metabolic process, iron-sulfur cluster assembly, oxidation-reduction process, ubiquinone activity); 3) urea catabolic process; 4) methionine metabolism; 5) iron ion binding; and 6) quorum sensing (data not shown).

\section{Markers of Gut Damage and Inflammation}

Next we measured circulating biomarkers of enterocyte turnover, microbial translocation, inflammation, and immune activation (Figure 5 and Supplementary Figure 2). Zonulin increased significantly between weeks 0 and 6: from $27.2 \mathrm{ng} / \mathrm{mL}$ at week 0 to $31.7 \mathrm{ng} / \mathrm{mL}$ at week $6(P=0.03$; Figure 5A), but no other biomarker changed significantly. PID3 had the highest zonulin, I-FABP, sTNFRI, sTNFRII, sTNFRSF8 (sCD30), and osteopontin levels of the participants and among the highest sCD14, sCD163, and (1,3)- $\beta$-D-glucan levels but the lowest levels of gp130, IL-6Ra, IL-8, osteocalcin, and TWEAK at week 0 (Figure 5 and Supplementary Figure 2). PID3's I-FABP and sTNFRSF 8 levels decreased by $>50 \%$ between weeks 0 and 6 (Figures 5B and 5D). Of note, 
higher I-FABP levels tended to correlate with lower $\alpha$ diversity at week $0(\mathrm{r}=-0.77, P=0.10)$ and at all time points combined $(\mathrm{r}=-0.59, P=0.01)$. Lower $\alpha$ diversity also correlated with lower IFN $\gamma$ $(\mathrm{r}=0.97, P=0.01)$ and MMP-1 levels $(\mathrm{r}=0.85, P=0.04)$ at week 0 and lower $\operatorname{IFN} \gamma(\mathrm{r}=0.53, P=0.02)$ and MMP-1 $(\mathrm{r}=0.61, P=0.008)$ and higher $\mathrm{SCD} 30(\mathrm{r}=-0.60, P=0.008)$ and $\mathrm{IL}-20(\mathrm{r}=-0.52, P=0.03)$ levels at all time points combined.

A

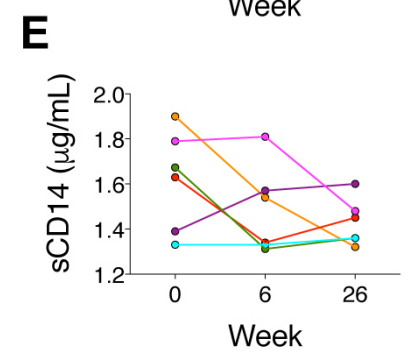

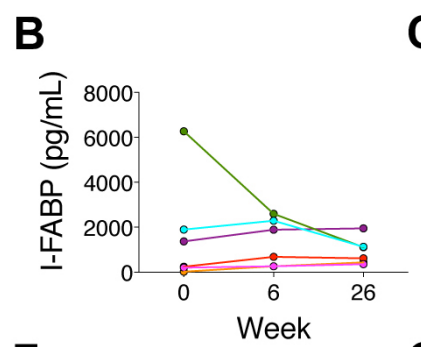

$\mathbf{F}$

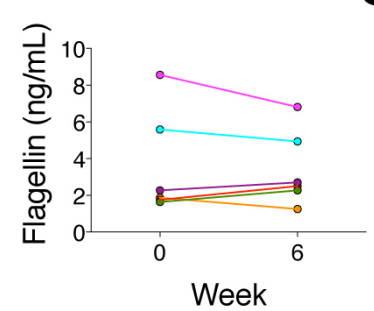

C

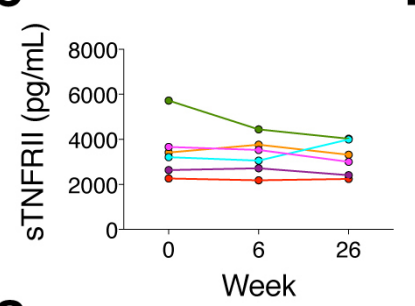

G

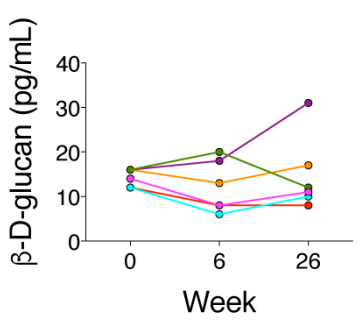

D

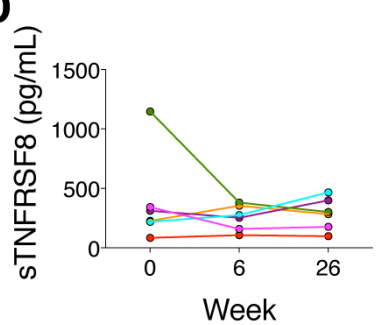

-0- PID6

- PID5

- PID4

$-0-$ PID3

- - PID2

- - PID1

Figure 5. Changes in biomarkers of gut damage (zonulin, intestinal fatty acid binding protein [I-FABP]), microbial translocation (soluble CD14 [sCD14], flagellin, and (1,3)- $\beta$-D-glucan), and inflammation (soluble tumor necrosis factor [TNF] receptor II [sTNFRII], and soluble TNF receptor superfamily 8 [sTNFRSF8]).

\section{Anthropomorphics and Hepatic Steatosis}

Weight did not change significantly between weeks 0 and 6 (median 0.5 kilograms [range -5 to 1.6; $P=0.75$ ) or between weeks 6 and 26 (median 0.7 kilograms [range -0.5 to $1.4 ; P=0.13$ ]). Waist circumference changed by a median of $-2.8 \mathrm{~cm}$ (range -5.0 to $2.0 ; P=0.19$ ) between weeks 0 and 6 and $-0.3 \mathrm{~cm}$ (range -4.0 to $3.3 ; P=0.81$ ) between weeks 6 and 26 . We found no significant change in hepatic steatosis between weeks 0 and 26 based on transient elastography with controlled attenuation parameter ( 278 [range 212 to 345 ] and 276 [range 201 to 300 ] $\mathrm{dB} / \mathrm{m}, P=0.91$ ).

\section{Confounding Factors}

PID2 received amoxicillin for an upper respiratory tract infection and clindamycin and levofloxacin just before week 26 for cellulitis. PID4 switched ART to bictegravir/tenofovir alafenamide/ emtricitabine in week 2, as did PID5 and PID6 between weeks 6 and 26. PID6 was also given clindamycin and ceftriaxone for an abscess in week 3. 


\section{DISCUSSION}

PWH have increased systemic inflammation, which drives end-organ events. In this pilot study, we administered weekly oral FMT with the goal of assessing safety and tolerability. We evaluated changes in dysbiosis and inflammation during and after the treatment period. We found that 1) oral FMT was safe and well-tolerated in PWH;2) $\alpha$ diversity increased between weeks 0 and 6 in PWH with low $\alpha$ diversity pre-FMT, but this change did not persist between weeks 6 and 26; 3 ) microbiome populations shifted towards the distribution of the donors between weeks 0 and 6, but no participant had complete engraftment of the FMT; and 4) one participant who had the lowest $\alpha$ diversity and highest I-FABP, zonulin, sTNFRI, sTNFRII, and TNFRSF8 levels at baseline had the greatest shift in microbiome distribution between weeks 0 and 6, with increases in bacteria that produce butyrate and SCFAs, decreases in bacteria of oral origin, and decreases in I-FABP, sCD14, sTNFRI, sTNFRII, TNFRSF8, and IL-6 levels.

The absence of complete engraftment is consistent with findings in other disease states such as $C$. difficile disease and IBD, where there is not complete replacement of the recipient's microbiome with the donor's microbiome, yet clinical benefits and changes in the microbiome with FMT have been observed [28-30]. This suggests that the effects of the microbiome may not be dependent upon a single organism or group of organisms but rather on the functionality of the microbiome. The participant with the greatest dysbiosis had the most significant changes in his microbiome and inflammatory markers, but it cannot be concluded definitively that these changes were due to FMT in the absence of a randomized, controlled trial. Nonetheless, whether inducing dysbiosis such as with antimicrobial agents will facilitate greater engraftment in more recipients is unknown. The development of appropriate metrics of desired functionality is needed so as to better evaluate the influences of microbiome composition and engraftment.

Alternatively, the bacteria and their functionality may be less important than other factors that are also transmitted, such as phages, SCFAs, and bile acids $[\underline{31}, \underline{32}]$. We observed changes in phages in PID3 but no consistent changes overall. SCFAs such as butyrate are crucial energy sources for colonic epithelial cells and tight junction maintenance and therefore critical for gut barrier integrity [33]. SCFAs also modulate neutrophil trafficking, stimulate regulatory $\mathrm{CD} 4^{+} \mathrm{T}$ cell generation, and decrease pro-inflammatory cytokine production by monocytes and macrophages [34]. Gut bacteria such as from Lachnospiraceae and Ruminococcaceae families convert primary bile acids, originating from the liver, into secondary bile acids [ㅌ5]. Bile acids, in turn, shape the composition of the gut microbiota. Thus, transmission of secondary bile acids may shape the recipient's microbiome independent of bacteria that are also transmitted.

Levels of $(1,3)-\beta$-D-glucan [36], sCD14 []] , and sCD163 [37] were on par with levels observed in people who are HIV-negative, whereas I-FABP and zonulin were consistent with other studies of PWH $[\underline{3}, \underline{38}]$. Together, these findings suggest that while enterocyte and tight junction turnover may be increased, microbial translocation and its ensuing inflammation was minimal in these participants. Thus, these participants may not have needed any intervention. T cell activation, reflected by sTNFRI, sTNFRII, and sTNFRSF8 (sCD30) [39], was high in the participant who had the most dramatic microbiome shift, but these levels declined between weeks 0 and 6 . It is tempting to speculate that PWH with high microbial translocation, immune activation, and systemic inflammation at baseline would garner the greatest benefit from FMT, but larger controlled studies of FMT for such a population are needed to determine whether that is the case. 
The low-level HIV viremia observed in several participants after starting FMT was unexpected. This may have been due to suboptimal adherence, but raises the question of whether FMT stimulated local inflammation in the gut, where most of the virus resides [눙, prompting $\mathrm{CD} 4^{+} \mathrm{T}$ cell activation and HIV RNA production or release from apoptotic cells. The increase in zonulin between weeks 0 and 6 may indicate more tight junction damage or turnover that could be consistent with local inflammation or, alternatively, the increase could reflect improved gut barrier function and less suppression of zonulin production. Systemic inflammation was not increased, and enterocyte turnover did not seem to change based on I-FABP levels. Nonetheless, this transient HIV viremia suggests that HIV RNA levels should be monitored closely in future FMT studies, and gut biopsies may be needed to elucidate the direct effects of FMT on gut integrity and inflammation.

Our study has several limitations. First, the study was small, with numerous confounding factors, and therefore underpowered to detect significant changes in the microbiome. The large number of analyses including the large number of pathways evaluated may have increased the risk of false positive findings in this small sample size. Second, the study lacked a placebo control, so it is challenging to conclude which changes are due to FMT versus which might have happened regardless. Third, the donors were selected based on the safety and efficacy of their stool for treating $C$. difficile patients, which may not translate to efficacy in PWH. In addition, engraftment may be donor-dependent [41]. Fourth, each recipient only received FMT from one donor; combining the microbiota of several healthy people into each FMT may increase the likelihood of a response. Fifth, neither the donors nor the recipients were counseled on a "microbiome-friendly diet" to maximize the likelihood of a healthy microbiome engrafting, and dietary histories were not collected. Lastly, most of the recipients were relatively healthy, with low levels of inflammation biomarkers, and half had $\alpha$ diversity on par with the donors'; therefore, they may not have been the population most likely to benefit. Rather, PWH with increased levels of inflammatory biomarkers and low $\alpha$ diversity may be most likely to benefit from FMT.

\section{CONCLUSION}

In summary, we found that administering multiple oral FMT was safe and well-tolerated. The changes in the microbiome during the treatment phase in some participants encourage us to pursue this line of research with enhanced methods of FMT treatment. Characteristics of an ideal oral FMT product are unknown, as are the optimal dose and treatment schedule. We are now combining the microbiota product of 3 donors, administered twice daily for 12 weeks, to increase the likelihood of significant engraftment in our studies of FMT in other chronic diseases. Whether oral FMT can decrease systemic inflammation in PWH with high baseline inflammation and low $\alpha$ diversity requires larger, controlled studies.

\section{SUPPLEMENTARY DATA}

Supplementary materials are available at the Pathogens and Immunity website. Supplementary data may be provided by the authors to benefit the reader. Supplementary data are not copyedited and are the sole responsibility of the authors. Questions or comments related to supplementary materials should be addressed to the corresponding author.

\section{Supplementary Figure 1 \\ Supplementary Figure 2}




\section{POTENTIAL CONFLICTS OF INTEREST}

H.L.D. and Z-D.J. have applied for a patent for PRIM-DJ2727, and H.L.D. has received a grant from Rebiotix to study their FMT product. MF is an employee of Associates of Cape Cod, Inc., the manufacturer of the $(1,3)-\beta$-D-glucan test used in this study. No other authors have competing interests.

\section{FUNDING}

This work was supported in by the Kelsey Research Foundation and by institutional funds from McGovern Medical School at The University of Texas Health Science Center at Houston.

\section{REFERENCES}

1. Utay NS, Overton ET. Immune activation and inflammation in people with HIV: Challenging targets. J Infect Dis. 2019. doi: 10.1093/infdis/jiz351. PubMed PMID: 31282534 .

2. Sandler NG, Douek DC. Microbial translocation in HIV infection: causes, consequences and treatment opportunities. Nat Rev Microbiol. 2012;10(9):655-66. Epub 2012/08/14. doi: 10.1038/nrmicro2848. PubMed PMID: 22886237.

3. Sandler NG, Wand H, Roque A, Law M, Nason MC, Nixon DE, Pedersen C, Ruxrungtham K, Lewin SR, Emery S, Neaton JD, Brenchley JM, Deeks SG, Sereti I, Douek DC. Plasma levels of soluble CD14 independently predict mortality in HIV infection. J Infect Dis. 2011;203(6):780-90. Epub 2011/01/22. doi: 10.1093/infdis/jiq118. PubMed PMID: 21252259; PMCID: Pmc3071127.

4. Tenorio AR, Zheng Y, Bosch RJ, Krishnan S, Rodriguez B, Hunt PW, Plants J, Seth A, Wilson CC, Deeks SG, Lederman MM, Landay AL. Soluble markers of inflammation and coagulation but not T-cell activation predict non-AIDS-defining morbid events during suppressive antiretroviral treatment. J Infect Dis. 2014;210(8):1248-59. Epub 2014/05/06. doi: 10.1093/infdis/jiu254. PubMed PMID: 24795473; PMCID: PMC4192039.

5. Hunt PW, Sinclair E, Rodriguez B, Shive C, Clagett B, Funderburg N, Robinson J, Huang Y, Epling L, Martin JN, Deeks SG, Meinert CL, Van Natta ML, Jabs DA, Lederman MM. Gut epithelial barrier dysfunction and innate immune activation predict mortality in treated HIV infection. J Infect Dis. 2014;210(8):1228-38. Epub 2014/04/24. doi: 10.1093/infdis/jiu238. PubMed PMID: 24755434; PMCID: PMC4192038.

6. Vujkovic-Cvijin I, Somsouk M. HIV and the Gut Microbiota: Composition, Consequences, and Avenues for Amelioration. Curr HIV/AIDS Rep. 2019;16(3):204-13. Epub 2019/05/01. doi: 10.1007/s11904-019-00441-w. PubMed PMID: 31037552; PMCID: PMC6579656.

7. Neff CP, Krueger O, Xiong K, Arif S, Nusbacher N, Schneider JM, Cunningham AW, Armstrong A, Li S, McCarter MD, Campbell TB, Lozupone CA, Palmer BE. Fecal Microbiota Composition Drives Immune Activation in HIV-infected Individuals. EBioMedicine. 2018;30:192-202. Epub 2018/04/14. doi: 10.1016/j.ebiom.2018.03.024. 
PubMed PMID: 29650491; PMCID: PMC5952409.

8. Li SX, Sen S, Schneider JM, Xiong KN, Nusbacher NM, Moreno-Huizar N, Shaffer M, Armstrong AJS, Severs E, Kuhn K, Neff CP, McCarter M, Campbell T, Lozupone CA, Palmer BE. Gut microbiota from high-risk men who have sex with men drive immune activation in gnotobiotic mice and in vitro HIV infection. PLoS Pathog. 2019;15(4):e1007611. Epub 2019/04/05. doi: 10.1371/journal.ppat.1007611. PubMed PMID: 30947289; PMCID: PMC6448819.

9. Noguera-Julian M, Rocafort M, Guillen Y, Rivera J, Casadella M, Nowak P, Hildebrand F, Zeller G, Parera M, Bellido R, Rodriguez C, Carrillo J, Mothe B, Coll J, Bravo I, Estany C, Herrero C, Saz J, Sirera G, Torrela A, Navarro J, Crespo M, Brander C, Negredo E, Blanco J, Guarner F, Calle ML, Bork P, Sonnerborg A, Clotet B, Paredes R. Gut Microbiota Linked to Sexual Preference and HIV Infection. EBioMedicine. 2016;5:13546. Epub 2016/04/15. doi: 10.1016/j.ebiom.2016.01.032. PubMed PMID: 27077120; PMCID: PMC4816837.

10. Kelley CF, Kraft CS, de Man TJ, Duphare C, Lee HW, Yang J, Easley KA, Tharp GK, Mulligan MJ, Sullivan PS, Bosinger SE, Amara RR. The rectal mucosa and condomless receptive anal intercourse in HIV-negative MSM: implications for HIV transmission and prevention. Mucosal Immunol. 2017;10(4):996-1007. Epub 2016/11/17. doi: 10.1038/mi.2016.97. PubMed PMID: 27848950; PMCID: PMC5433931.

11. Armstrong AJS, Shaffer M, Nusbacher NM, Griesmer C, Fiorillo S, Schneider JM, Preston Neff C, Li SX, Fontenot AP, Campbell T, Palmer BE, Lozupone CA. An exploration of Prevotella-rich microbiomes in HIV and men who have sex with men. Microbiome. 2018;6(1):198. Epub 2018/11/07. doi: 10.1186/s40168-018-0580-7. PubMed PMID: 30396369; PMCID: PMC6219090.

12. McDonald LC, Gerding DN, Johnson S, Bakken JS, Carroll KC, Coffin SE, Dubberke ER, Garey KW, Gould CV, Kelly C, Loo V, Shaklee Sammons J, Sandora TJ, Wilcox MH. Clinical Practice Guidelines for Clostridium difficile Infection in Adults and Children: 2017 Update by the Infectious Diseases Society of America (IDSA) and Society for Healthcare Epidemiology of America (SHEA). Clin Infect Dis. 2018;66(7):e1e48. Epub 2018/02/21. doi: 10.1093/cid/cix1085. PubMed PMID: 29462280; PMCID: PMC6018983.

13. Vujkovic-Cvijin I, Rutishauser RL, Pao M, Hunt PW, Lynch SV, McCune JM, Somsouk M. Limited engraftment of donor microbiome via one-time fecal microbial transplantation in treated HIV-infected individuals. Gut Microbes. 2017;8(5):440-50. Epub 2017/05/26. doi: 10.1080/19490976.2017.1334034. PubMed PMID: 28541799; PMCID: PMC5628639.

14. Ding X, Li Q, Li P, Zhang T, Cui B, Ji G, Lu X, Zhang F. Long-Term Safety and Efficacy of Fecal Microbiota Transplant in Active Ulcerative Colitis. Drug Saf. 2019;42(7):86980. Epub 2019/04/12. doi: 10.1007/s40264-019-00809-2. PubMed PMID: 30972640.

15. Moayyedi P, Surette MG, Kim PT, Libertucci J, Wolfe M, Onischi C, Armstrong D, Marshall JK, Kassam Z, Reinisch W, Lee CH. Fecal Microbiota Transplantation Induces Remission in Patients With Active Ulcerative Colitis in a Randomized Controlled 
Trial. Gastroenterology. 2015;149(1):102-9 e6. Epub 2015/04/11. doi: 10.1053/j.gastro.2015.04.001. PubMed PMID: 25857665.

16. Jiang ZD, Jenq RR, Ajami NJ, Petrosino JF, Alexander AA, Ke S, Iqbal T, DuPont AW, Muldrew K, Shi Y, Peterson C, Do KA, DuPont HL. Safety and preliminary efficacy of orally administered lyophilized fecal microbiota product compared with frozen product given by enema for recurrent Clostridium difficile infection: A randomized clinical trial. PLoS One. 2018;13(11):e0205064. Epub 2018/11/06. doi: 10.1371/journal. pone.0205064. PubMed PMID: 30388112; PMCID: PMC6214502.

17. Glass RI, Noel J, Ando T, Fankhauser R, Belliot G, Mounts A, Parashar UD, Bresee JS, Monroe SS. The epidemiology of enteric caliciviruses from humans: a reassessment using new diagnostics. J Infect Dis. 2000;181 Suppl 2:S254-61. Epub 2000/05/11. doi: 10.1086/315588. PubMed PMID: 10804134.

18. Ottesen A, Ramachandran P, Reed E, White JR, Hasan N, Subramanian P, Ryan G, Jarvis K, Grim C, Daquiqan N, Hanes D, Allard M, Colwell R, Brown E, Chen Y. Enrichment dynamics of Listeria monocytogenes and the associated microbiome from naturally contaminated ice cream linked to a listeriosis outbreak. BMC Microbiol. 2016;16(1):275. Epub 2016/11/18. doi: 10.1186/s12866-016-0894-1. PubMed PMID: 27852235; PMCID: PMC5112668.

19. Ponnusamy D, Kozlova EV, Sha J, Erova TE, Azar SR, Fitts EC, Kirtley ML, Tiner BL, Andersson JA, Grim CJ, Isom RP, Hasan NA, Colwell RR, Chopra AK. Cross-talk among flesh-eating Aeromonas hydrophila strains in mixed infection leading to necrotizing fasciitis. Proc Natl Acad Sci U S A. 2016;113(3):722-7. Epub 2016/01/07. doi: 10.1073/pnas.1523817113. PubMed PMID: 26733683; PMCID: PMC4725515.

20. Hasan NA, Young BA, Minard-Smith AT, Saeed K, Li H, Heizer EM, McMillan NJ, Isom R, Abdullah AS, Bornman DM, Faith SA, Choi SY, Dickens ML, Cebula TA, Colwell RR. Microbial community profiling of human saliva using shotgun metagenomic sequencing. PLoS One. 2014;9(5):e97699. Epub 2014/05/23. doi: 10.1371/journal. pone.0097699. PubMed PMID: 24846174; PMCID: PMC4028220.

21. Lax S, Smith DP, Hampton-Marcell J, Owens SM, Handley KM, Scott NM, Gibbons SM, Larsen P, Shogan BD, Weiss S, Metcalf JL, Ursell LK, Vazquez-Baeza Y, Van Treuren W, Hasan NA, Gibson MK, Colwell R, Dantas G, Knight R, Gilbert JA. Longitudinal analysis of microbial interaction between humans and the indoor environment. Science. 2014;345(6200):1048-52. Epub 2014/08/30. doi: 10.1126/science.1254529. PubMed PMID: 25170151; PMCID: PMC4337996.

22. Li D, Liu CM, Luo R, Sadakane K, Lam TW. MEGAHIT: an ultra-fast single-node solution for large and complex metagenomics assembly via succinct de Bruijn graph. Bioinformatics. 2015;31(10):1674-6. Epub 2015/01/23. doi: 10.1093/bioinformatics/ btv033. PubMed PMID: 25609793.

23. Seemann T. Prokka: rapid prokaryotic genome annotation. Bioinformatics. 2014;30(14):2068-9. Epub 2014/03/20. doi: 10.1093/bioinformatics/btu153. PubMed PMID: 24642063.

24. Reichardt N, Duncan SH, Young P, Belenguer A, McWilliam Leitch C, Scott KP, Flint 
HJ, Louis P. Phylogenetic distribution of three pathways for propionate production within the human gut microbiota. ISME J. 2014;8(6):1323-35. Epub 2014/02/21. doi: 10.1038/ismej.2014.14. PubMed PMID: 24553467; PMCID: PMC4030238.

25. Fu X, Liu Z, Zhu C, Mou H, Kong Q. Nondigestible carbohydrates, butyrate, and butyrate-producing bacteria. Crit Rev Food Sci Nutr. 2019;59(sup1):S130-S52. Epub 2018/12/26. doi: 10.1080/10408398.2018.1542587. PubMed PMID: 30580556.

26. Staley C, Weingarden AR, Khoruts A, Sadowsky MJ. Interaction of gut microbiota with bile acid metabolism and its influence on disease states. Appl Microbiol Biotechnol. 2017;101(1):47-64. Epub 2016/11/27. doi: 10.1007/s00253-016-8006-6. PubMed PMID: 27888332; PMCID: PMC5203956.

27. Weingarden AR, Chen C, Bobr A, Yao D, Lu Y, Nelson VM, Sadowsky MJ, Khoruts A. Microbiota transplantation restores normal fecal bile acid composition in recurrent Clostridium difficile infection. Am J Physiol Gastrointest Liver Physiol. 2014;306(4):G310-9. Epub 2013/11/29. doi: 10.1152/ajpgi.00282.2013. PubMed PMID: 24284963 ; PMCID: PMC3920123.

28. Staley C, Kaiser T, Vaughn BP, Graiziger CT, Hamilton MJ, Rehman TU, Song K, Khoruts A, Sadowsky MJ. Predicting recurrence of Clostridium difficile infection following encapsulated fecal microbiota transplantation. Microbiome. 2018;6(1):166. Epub 2018/09/20. doi: 10.1186/s40168-018-0549-6. PubMed PMID: 30227892; PMCID: PMC6145197.

29. Zou M, Jie Z, Cui B, Wang H, Feng Q, Zou Y, Zhang X, Yang H, Wang J, Zhang F, Jia $\mathrm{H}$. Fecal microbiota transplantation results in bacterial strain displacement in patients with inflammatory bowel diseases. FEBS Open Bio. 2020;10(1):41-55. Epub 2019/10/18. doi: 10.1002/2211-5463.12744. PubMed PMID: 31622538; PMCID: PMC6943227.

30. Hirten RP, Grinspan A, Fu SC, Luo Y, Suarez-Farinas M, Rowland J, Contijoch EJ, Mogno I, Yang N, Luong T, Labrias PR, Peter I, Cho JH, Sands BE, Colombel JF, Faith JJ, Clemente JC. Microbial Engraftment and Efficacy of Fecal Microbiota Transplant for Clostridium Difficile in Patients With and Without Inflammatory Bowel Disease. Inflamm Bowel Dis. 2019;25(6):969-79. Epub 2019/03/11. doi: 10.1093/ibd/izy398. PubMed PMID: 30852592; PMCID: PMC6499938.

31. DuPont HL, Jiang ZD, DuPont AW, Utay NS. Abnormal Intestinal Microbiome in Medical Disorders and Potential Reversibility by Fecal Microbiota Transplantation. Dig Dis Sci. 2020;65(3):741-56. Epub 2020/02/03. doi: 10.1007/s10620-020-06102-y. PubMed PMID: 32008133.

32. Ott SJ, Waetzig GH, Rehman A, Moltzau-Anderson J, Bharti R, Grasis JA, Cassidy L, Tholey A, Fickenscher H, Seegert D, Rosenstiel P, Schreiber S. Efficacy of Sterile Fecal Filtrate Transfer for Treating Patients With Clostridium difficile Infection. Gastroenterology. 2017;152(4):799-811 e7. Epub 2016/11/22. doi: 10.1053/j.gastro.2016.11.010. PubMed PMID: 27866880.

33. Yu LC, Wang JT, Wei SC, Ni YH. Host-microbial interactions and regulation of intestinal epithelial barrier function: From physiology to pathology. World J Gastrointest 
Pathophysiol. 2012;3(1):27-43. Epub 2012/03/01. doi: 10.4291/wjgp.v3.i1.27. PubMed PMID: 22368784; PMCID: PMC3284523.

34. Correa-Oliveira R, Fachi JL, Vieira A, Sato FT, Vinolo MA. Regulation of immune cell function by short-chain fatty acids. Clin Transl Immunology. 2016;5(4):e73. Epub 2016/05/20. doi: 10.1038/cti.2016.17. PubMed PMID: 27195116; PMCID: PMC4855267.

35. Baktash A, Terveer EM, Zwittink RD, Hornung BVH, Corver J, Kuijper EJ, Smits WK. Mechanistic Insights in the Success of Fecal Microbiota Transplants for the Treatment of Clostridium difficile Infections. Front Microbiol. 2018;9:1242. Epub 2018/06/28. doi: 10.3389/fmicb.2018.01242. PubMed PMID: 29946308 ; PMCID: PMC6005852.

36. Pruller F, Wagner J, Raggam RB, Hoenigl M, Kessler HH, Truschnig-Wilders M, Krause R. Automation of serum (1-->3)-beta-D-glucan testing allows reliable and rapid discrimination of patients with and without candidemia. Med Mycol. 2014;52(5):455-61. Epub 2014/06/08. doi: 10.1093/mmy/myu023. PubMed PMID: 24906361.

37. Burdo TH, Lentz MR, Autissier P, Krishnan A, Halpern E, Letendre S, Rosenberg ES, Ellis RJ, Williams KC. Soluble CD163 made by monocyte/macrophages is a novel marker of HIV activity in early and chronic infection prior to and after anti-retroviral therapy. J Infect Dis. 2011;204(1):154-63. Epub 2011/06/02. doi: 10.1093/infdis/jir214. PubMed PMID: 21628670; PMCID: PMC3105035.

38. Utay NS, Somasunderam A, Hinkle JE, Petschow BW, Detzel CJ, Somsouk M, Fichtenbaum CJ, Weaver EM, Shaw AL, Asmuth DM. Serum Bovine Immunoglobulins Improve Inflammation and Gut Barrier Function in Persons with HIV and Enteropathy on Suppressive ART. Pathog Immun. 2019;4(1):124-46. Epub 2019/05/30. doi: 10.20411/pai.v4i1.276. PubMed PMID: 31139758; PMCID: PMC6508431.

39. Ward-Kavanagh LK, Lin WW, Sedy JR, Ware CF. The TNF Receptor Superfamily in Co-stimulating and Co-inhibitory Responses. Immunity. 2016;44(5):1005-19. Epub 2016/05/19. doi: 10.1016/j.immuni.2016.04.019. PubMed PMID: 27192566; PMCID: PMC4882112.

40. Estes JD, Kityo C, Ssali F, Swainson L, Makamdop KN, Del Prete GQ, Deeks SG, Luciw PA, Chipman JG, Beilman GJ, Hoskuldsson T, Khoruts A, Anderson J, Deleage C, Jasurda J, Schmidt TE, Hafertepe M, Callisto SP, Pearson H, Reimann T, Schuster J, Schoephoerster J, Southern P, Perkey K, Shang L, Wietgrefe SW, Fletcher CV, Lifson JD, Douek DC, McCune JM, Haase AT, Schacker TW. Defining total-body AIDS-virus burden with implications for curative strategies. Nat Med. 2017;23(11):1271-6. Epub 2017/10/03. doi: 10.1038/nm.4411. PubMed PMID: 28967921; PMCID: PMC5831193.

41. Wilson BC, Vatanen T, Cutfield WS, O'Sullivan JM. The Super-Donor Phenomenon in Fecal Microbiota Transplantation. Front Cell Infect Microbiol. 2019;9:2. Epub 2019/02/06. doi: 10.3389/fcimb.2019.00002. PubMed PMID: 30719428; PMCID: PMC6348388. 


\section{FOOTNOTES}

Submitted June 8, 2020 | Accepted November 13, 2020 | Published December 30, 2020

\section{COPYRIGHT}

Copyright (C) 2020 Pathogens and Immunity. This is an open-access article distributed under the terms of the Creative Commons Attribution 4.0 International License. 